\title{
La prise de la parole dans Les Contes philosophiques de Diderot
}

\section{Primož Vitez}

\section{Résumé}

Tous les contes de Diderot, et la plupart de ses romans, fonctionnent comme du théâtre latent. En outre, Diderot se lit comme un auteur qui a réussi à unir en sa personne le don de dire de la philosophie et celui de raconter des histoires. Il y a plus d'une voie pour un humaniste de parvenir à une interprétation du monde social. Certains textes de Diderot sont explicitement le fruit d'une réflexion philosophique, d'autres excellent en un artisme authentique - ce qui a valu à Diderot la réputation de l'un des écrivains les plus dialogiques du dix-huitième siècle européen. Les deux énergies créatrices se trouvent réunies dans ses courtes histoires, écrites entre 1768 et 1774 . Ces textes s'entendent comme philosophiques parce qu'ils s'ouvrent sur des dilemmes éthiques fondamentaux: ils se destinent à donner la parole à l'individu quand celui-ci se trouve confronté à l'arbitraire de la loi ou à celui de l'opinion publique.

Mots-clés: prise de la parole, Diderot, éthique de la parole, dialogue, voix littéraire 
Le conte, pour Diderot, est un spectacle narratif. Faire des contes, en français classique, relève de l'omniprésente culture conversationnelle, soigneusement élaborée parmi les intellectuels, c'est-à-dire surtout dans des salons de l'aristocratie et de la haute bourgeoisie ou se forgeait, à partir du début du XVII ${ }^{\mathrm{e}}$ siècle, la norme écrite et parlée de la langue française. Pour lire les contes de Diderot, ${ }^{1}$ il est indispensable de comprendre le contexte de leur dimension parlée; ce qui n'est pas difficile, puisque ces textes, en général, sont écrits comme des transcriptions de dialogues que l'auteur prétend avoir réellement vécu (Attali 2012: 356). Son coup de génie, c'est de présenter ces dialogues comme s'ils étaient proférés par des gens de théâtre, par des comédiens. Cela ne devrait étonner personne: Diderot est l'auteur-clé à qui voudrait comprendre la nature du théâtre français au dix-huitième siècle. Ses textes explicitement dramatiques n'ont plus, à vrai dire, le potentiel scénique qui attirerait souvent les gens de théâtre à les réaliser; mais ses contes philosophiques, écrits sous forme de dialogues, sont imprégnés d'une irrécusable sensibilité dramatique formant des situations et des conflits psychologiques qu'il vaudrait absolument la peine, aujourd'hui, de réaliser sur scène. L'intention littéraire, chez Diderot, vibre en pulsions orales. ${ }^{2} \mathrm{Du}$ reste, il a déployé le meilleur de son effort littéraire pour écrire de la parole.

Son premier conte sous forme de récit bref, plus ou moins anecdotique, $M y s t i-$ fication, Diderot l'a écrit à l'âge de cinquante-six ans. Sa sensibilité philosophique et artistique était donc bien élaborée; à l'époque il avait déjà écrit la plupart des ouvrages qui ont illustré son nom: trois romans, les principaux traités philosophiques, mais il avait surtout terminé, en duo avec d'Alembert, l'exploit de sa vie, la monumentale Encyclopédie (1747-1766) qui a résumé les Lumières à l'usage de toute l'Europe et établi de nouveaux standards dans la formulation et organisation de la connaissance. Cela peut paraître surprenant, mais Diderot, comme Voltaire ${ }^{3}$ avant lui, s'est d'abord attaqué aux grandes formes littéraires et philosophiques pour passer enfin à la moins prestigieuse, celle du court récit en prose.

Diderot est un auteur qui développe soigneusement la complexité de son énonciation littéraire. Non seulement il déploie devant le lecteur le dynamisme des couches narratives de ses récits, il n'hésite pas de lui fournir au fur et à mesure

1 Toutes les notes citant les extraits des contes de Diderot, sauf exception notée, se référeront à l'excellente édition critique, publiée par Laurent Versini: Diderot, Oeuvres, tome II, Contes, Paris: Robert Laffont, 1994.

2 Dans la Lettre sur les sourds et muets, Diderot spécule sur l'origine de la parole mettant en valeur l'absence des fonctions phonatoires, car 'un sourd-muet de naissance est sans préjugé sur la manière de communiquer sa pensée.' Par ce biais privatif, il postule implicitement que la phonation se trouve à la source des conventions sociales, la Lettre étant adressée 'à l'usage de ceux qui entendent E̊ qui parlent'.

3 Voltaire publie Candide en 1759, à l'âge de soixante-cinq ans, après avoir écrit des dizaines de tragédies en alexandrins. 
des explications poétologiques de son activité littéraire. Dans Les deux amis de Bourbonne (1770), il dévoile son approche poétique dans le corps même du conte. Au moment du dénouement de l'histoire, Diderot explique qu'il classe ses écrits brefs sous le genre de conte historique qu'il définit minutieusement :

[L'auteur] se propose de vous tromper; il est assis au coin de votre âtre; il a pour objet la vérité rigoureuse; il veut être cru; il veut intéresser, toucher, entraîner, émouvoir, faire frissonner la peau et couler les larmes; effet qu'on n'obtient point sans éloquence et sans poésie. Mais l'éloquence est une sorte de mensonge, et rien de plus contraire à l'illusion que la poésie; l'une et l'autre exagèrent, surfont, amplifient, inspirent la méfiance: comment s'y prendra donc ce conteur-ci pour vous tromper? Le voici. Il parsèmera son récit de petites circonstances si liées à la chose, de traits si simples, si naturels, et toutefois si difficiles à imaginer, que vous serez forcé de vous dire en vous-même: $\mathrm{Ma}$ foi, cela est vrai: on n'invente pas ces choses-là. C'est ainsi qu'il sauvera l'exagération de l'éloquence et de la poésie; que la vérité de la nature couvrira le prestige de l'art; et qu'il satisfera à deux conditions qui semblent contradictoires, d'être en même temps historien et poëte, véridique et menteur. ${ }^{4}$

Diderot, connaisseur et admirateur des beaux-arts, précise sa position en ajoutant un exemple, issu de la peinture. La réalité, ${ }^{5}$ dit-il, se laisse provoquer par le détail: l'artiste peut idéaliser ses motifs de façon à ce qu'on n'y découvrirait que des projections de la nature, parce qu'il les réalise en formes symétriques. S'il applique, en revanche, à cet idéal quelques petits signes de réalité, il en résultera une image que le spectateur pourra référer à son expérience personnelle. L'inaccessible effigie d'une divine Vénus, à qui on a ajouté une petite irrégularité de la peau, deviendra subitement le visage d'une belle voisine. Une image idéalisée se métamorphose ainsi en ce que le spectateur reconnaîtra comme naturel. Il cite à ce propos son ami Caillot, comédien:

Un peu de poussière sur mes souliers et je ne sors pas de ma loge, je reviens de la campagne. ${ }^{6}$

La dénomination poétique qu'adopte Diderot pour ses récits brefs, conte historique, ne signifie pas que le conteur choisit de parler d'une époque ou de personnages historiques particuliers. Le terme renvoie plutôt à l'intérêt que porte l'auteur

4 Les deux amis de Bourbonne, p. 480.

5 C'est, du point de vue analytique de l'Encyclopédie, le sens que prend la réflexion de Beauzée à propos de la langue en général, citée dans Sylvain Auroux, L'Encyclopédie: Grammaire et langue au XVIIIe siècle (Paris: Mame, 1973), p. 95: "Tout est usage dans les langues; le matériel et la signification des mots, l'analogie et l'anomalie des terminaisons, la servitude ou la liberté des constructions, le purisme ou le barbarisme des ensembles. [... L'usage nèst donc pas le tyran des langues, il en est le législateur naturel, nécessaire, et exclusif."

Les deux amis de Bourbonne, p. 481. 
pour les événements et détails réels dans une histoire, c'est-à-dire cette réalité foncièrement dissymétrique et véridique qui devrait pourtant bien intéresser l'historiographe quand il écrit de l'Histoire. Diderot, en racontant ce qui l'intéresse, historise l'événement sans intention de tailler des monuments.

Il ne s'agit pas de reproduire des échantillons formels que l'observateur est généralement capable d'extraire de son environnement, mais de développer le principe du détail qui produira la réalité de la fiction, qui s'adressera à celui qui écoute ou qui lit et qui traduira, dans le récit, l'imperfection, la fautivité, l'instabilité mais aussi la beauté de la nature et de l'homme. D'ailleurs, Mornet a bien esquissé l'esthétique littéraire chez Diderot:

Ce qu'il y a, si l'on veut, de réalisme dans ces contes de Diderot, c'est ce qu'on pourrait appeler le réalisme pittoresque. Nous n'y trouvons pas seulement des aventures, les actions et les réactions des caractères. Nous voyons les personnages s'y mouvoir, trahir leurs caractères par leurs gestes et leurs physionomies. (Mornet 1966: 135)

L'observation des faits naturels se concentre souvent au fonctionnement de la nature humaine ce qui amène nécessairement l'observateur à s'observer et à s'analyser lui-même. Son dévouement à la pensée n'entraîne pas de préjugés idéologiques: ses analyses n'imposent aucun parti pris interprétatif, au contraire, elles se proposent d'ouvrir, à la vue du lecteur, l'accès au problème traité. Par son expérience intellectuelle et quotidienne, il construit sans moraliser cet unique laboratoire moral qu'est son conte historique.

Le titre du texte central parmi ces contes, Ceci n'est pas un conte, se lit sous quelques aspects apparamment divergents. L'énoncé 'ceci n'est pas un conte' est d'abord une explication de l'auteur qui prétend n'avoir rien inventé; que les choses racontées sont, ni plus ni moins, celles qu'il avait réellement vécues et qu'il a l'intention de rendre telles quelles. C'est bien dans la rendition présumée des histoires 'telles quelles' qu'est forgée la finesse dramaturgique de Diderot. C'est une question de technique. D'un autre côté, l'auteur se propose d'expliquer que ses historiettes ne sont pas des contes conventionnels que le lecteur aurait pris l'habitude de lire. Diderot a consacré beaucoup de son énérgie créatrice à élaborer soigneusement des couches narratives dans ses récits ce qui lui a valu, à juste titre, la réputation de novateur dans la formulation de la raison en philosophie et en fiction. Enfin, en refusant de confirmer les attentes du lecteur, Diderot propose à son public une troisième interprétation, implicitement énoncée comme un avis ou conseil: lisez attentivement, car les conteurs aiment bien 'faire semblant' de raconter des fantaisies, alors qu'ils se servent en cachette de leur art littéraire pour créer l'illusion dans laquelle il semble devenir clair que 
leurs histoires sont les seules possibles, c'est-à-dire réelles. Il faut lire, paraît-il, ces contes dans les trois sens.

Pendant longtemps, jusqu'en 1954, quand il a été publié pour la première fois en France, le premier conte à compter aujourd'hui parmi ces traités fictionnels sur la morale, Mystification, était considéré comme une simple ébauche, comme un tronc littéraire, indigne de publication accomplie. Du point de vue du développement de l'histoire, c'est un texte qui peut paraître inachevé, puisque les événements s'arrêtent brusquement en privant le lecteur du dénouement narratif: l'histoire n'a pas de suite parce que le héros central, se mettant en scène comme un médecin turc pour duper une demoiselle crédule, selon le narrateur, s'est tiré réellement deux balles dans le cerveau ce qui laissait l'impression d'incomplétude de l'intrigue, autrement dit, de la mystification racontée. Mystification en tant que corps textuel, écrit par une majuscule, comporte tous les éléments essentiels de la littérature de Diderot: documentarité, donc référence à des événements réels et surtout le dialogue captant, marque ineffable de cette écriture théâtrale - malgré son apparence prosaïque; et puis le positionnement philosophique de Diderot qui savait mettre la même force argumentative dans les deux côtés de la controverse. Il donne la parole à chaque individu pour en tirer, sans prendre parti, des conséquences synthétiques relevant de la justice raisonnée.

Les textes littéraires de Diderot cherchaient des voies atypiques pour arriver à leur public. Les contes Deux amis de Bourbonne et Entretien d'un père avec ses enfants ont été écrits en quelques semaines en 1770, puis remis à un ami, nommé Melchior Grimm, éditeur de la Correspondance littéraire, fascicule périodique qui à l'époque n'avait qu'une quinzaine d'abonnées dont aucun en France. Cette publication était destinée aux princes et aristocrates des différentes cours européennes, désireux d'être au courant de ce qui se passait à Paris, en littérature française. Les textes, qui y étaient publiés, n'avaient certainement pas de public classique. Ces deux contes ont d'abord été publiés en 1772 à Zurich, incorporés dans un recueil de contes qu'écrivait Salomon Gessner, écrivain suisse. Ce n'est que l'année d'après que les deux textes étaient sortis en France. Ce détail rappelle fortement le destin du Neveu de Rameau qui a été publié pour la première fois en Allemagne, en 1805, donc longtemps après la mort de Diderot, et en traduction de Goethe, pour enfin paraître en France en 1821 - d'abord en traduction de l'allemand.

Entretien d'un père avec ses enfants est un conte typique de Diderot, dialogué et basé sur une soirée que Diderot, en compagnie de son frère Didier (qui était prêtre), de sa 'soeurette' et de quelques visiteurs, avait passée avec son père. Le conte thématise les rapports que l'homme établit avec les principes de réglementation sociale et civile: l'individu peut les formuler à travers son penchant pour l'intérêt personnel, ou bien à travers son sens intime de la justice, de la responsabilité sociale et du bien commun. D'ailleurs, le conte est sous-titré Du danger de se 
mettre au-dessus des lois et ouvre par là le débat entre le bon sens et les lois civiles, surtout quand celles-ci produisent de l'iniquité, des effets nuisibles ou injustes à la sensibilité du bien. Diderot, sous dénomination fictionnelle cette fois-ci de Philosophe ou Moi, se réclame de la culture du coeur et de la probité du jugement personnel, fondé sur l'expérience sociale, sur la loi morale intérieure et sur une certaine sagesse qui devrait, c'est son avis, s'imposer sur certains usages critiquables dans la société qui, dans leurs généralisations, sont souvent insensibles aux réalités sociales. Le père défend son légalisme en arguant que le jugement intime est corruptible et enclin à l'erreur, puisqu'il se fonde sur l'expérience particulière, étant ainsi sujet à l'intérêt particulier. En terminant le récit sur la soirée familiale, le personnage Moi se penche à l'oreille de son géniteur:

- Mon père, c'est qu'à la rigueur il n'y a point de loi pour le sage.

- Parlez plus bas.

- Toutes étant sujettes à des exceptions, c'est à lui qu'il appartient de juger des cas où il faut s'y soumettre ou s'en affranchir.

- Je ne serais pas trop fâché, me répondit-il, qu'il y eût dans la ville un ou deux citoyens comme toi, mais je n'y habiterais pas, s'ils pensaient tous de même. ${ }^{7}$

C'est le père qui a le droit au dernier mot et ce n'est pas un hasard. Le conte appartient au cycle que Diderot avait écrit lors du voyage à Langres pour retrouver l'attachement à la tradition de la parole paternelle. Et puis, bien sûr, la composante centrale de l'art de Diderot (ainsi que de sa puissance philosophique), c'est de savoir concilier, dans une très solide culture conversationnelle, des disputes apparamment inconciliables, en formulant les antagonismes par une même vigueur argumentative et stylistique. L'antagonisme idéologique peut être un processus efficace dans la formation du positionnement politique du locuteur, mais n'a d'habitude rien à voir avec la vérité des lieux-communs concernant la réalité, parce qu'en l'observant d'un peu plus près, on n'y découvre que du parti pris.

Dans la fiction documentaire de Diderot, Ceci n'est pas un conte, Madame de la Carlière et Supplément au Voyage de Bougainville constituent le bloc central, ayant acquis par la critique littéraire le dénominateur commun de cycle parisien. Il y est ostentativement question du temps estival, sans doute parce que ces trois contes ont été écrit pendant l'été 1772. Le discours météorologique, d'ailleurs, est une intervention originale de Diderot, non seulement parce qu'il exploite à merveille sa fonction phatique qui remplit les répliques de ceux qui au début de la conversation semblent tâtonner le sujet, mais surtout parce que ce discours est élevé au niveau scientifique - voire encyclopédique. Le temps qu'il fait n'est pas simplement

$7 \quad$ Entretien d'un père avec ses enfants, p. 502. 
beau ou mauvais: les personnages parlent de la dissolution des nébuleuses qui laissent entrevoir des éclaircies dans un ciel nuageux, de la condensation des micro-gouttes dans les vapeurs atmosphériques, de l'équilibre entre les pressions aériennes qui deviennent peu à peu des métaphores pour les rapports émotionnels entre les hommes et les femmes qui se construisent ou se défont par des altérations psycho-physiques, emportés par les vents de la passion, saturés par le bien ou le mal dans les températures changeantes pour se dénouer dans des tempêtes affectives ou des stagnations d'indifférence.

Le thème central de ces contes étant les relations (matrimoniales) entre homme et femme, Diderot est encore ici le 'traducteur' de sa propre vie: il mariait en 1772 sa fille unique, la bien-aimée Angélique, et c'est un mariage qui a profondément occupé ses soins et son esprit. Il avait élevé la jeune fille en une femme talentueuse, consciente de ses talents, intelligente et noblement sensible, habile en conversation intellectuelle, ses lectures allant bien au-delà du catéchisme de l'aprés-midi. Ceci n'est pas un conte est une combinaison de deux histoires parallèles où la tragédie amoureuse est tour à tour provoquée par une femme moralement corrompue ou par un homme sans vertu, leurs partenaires étant victimes de leur propre naïveté et bonté inconditionnelle. Comme nous avons déjà pu le constater par rapport aux textes précédents, l'auteur ne porte de jugement schématique sur aucun des profils moraux et psychologiques de ses personnages; il préfère élaborer une certaine ambivalence qui seule reconstruit l'image exacte du caractère. Les personnages sont écrits de façon à ce que leur (im)moralité soit lue dans la lumière des réactions caractérielles qui apparaissent au fur et à mesure du déroulement de l'histoire. Ainsi, on ne sait pas très bien si le pauvre Tanié est irréparablement bon ou tout simplement ridicule; on a du mal à décider si la terrible Reymer n'est qu'un monstre calculateur ou bien une femme réaliste qui avait appris à écarter toute indulgence par rapport au sexe privilégié. La grâcieuse Mademoiselle de la Chaux qui court à sa perdition en vouant complètement ses talents à un amant, n'est peut-être qu'une fille crédule, et l'orgueilleux Gardeil risque d'apparaître comme un bonvivant habile qui sait parfaitement organiser son entourage et créer l'impression que les efforts de ses proches n'arrangent pas seulement son bien, mais en même temps celui de l'intérêt commun. Diderot ne moralise pas: les nuances psychologiques, selon lesquelles il fait parler ses personnages, se tissent au fur et à mesure de l'emprise des dilemmes interprétatifs, comparables au dichotomies sémantiques où l'on a parfois du mal à poser la distinction entre avarice et économie, générosité et gaspillage, stupidité et confiance. Jouant les échelles de la vieille terminologie moraliste, l'auteur est assez intelligent pour ne pas imposer de jugements de valeur: il propose plutôt au lecteur, par son réflexe profondément humaniste, de résoudre tout seul le choix interprétatif et de lui entr'ouvrir la porte vers la relativité du jugement moral. 
Au moment du mariage d'Angélique, Diderot approfondit sa réflexion par rapport au statut social de la femme. La Reymer, Mademoiselle de la Chaux, mais surtout Madame de la Carlière, ne ressemblent nullement à la frivole Dornet de la Mystification, qui, deux ans auparavant sautillait d'amant à amant, et s'abandonnait à la superstition. Dans le monde masculin, la femme doit se constituer comme une intégrité psychologique et morale: elle doit devenir philosophe. Elle doit faire preuve d'une infinie débrouillardise dans la recherche d'un équilibre entre la stabilité prescrite par les lois civiles et religieuses et le dynamisme psychique et corporel - tel qu'il découle des réalités biologiques de la loi naturelle - et, si on veut - de l'inconstance du temps. Elle doit éviter les extrêmes et trouver son chemin entre le comique d'une bonté exagérée et la tragique manipulation, car, selon les exemples qu'on a pu vivre et lire, les deux sont garants de sa perte sociale et individuelle. Entre la légèreté débridée et une létargie immuable, la femme mariée doit s'aventurer sur ce qu'on appellerait la voie alternative qui, par le recours à la solidité des arguments moraux, lui garantirait le droit à la dignité humaine, le droit à la raison. Bref, elle doit apprendre à parler. Les sociétés oublient facilement que l'égalité est un droit de départ et non un objectif lointain que devrait poursuivre un antagoniste social dans sa lutte inégale. Le problème de la femme se range ainsi au centre de la réflexion morale de Diderot et devient prototypique de ses résolutions philosophiques: les extrêmes sont insuffisants, sans égard à leur sens, le fanatisme est inadmissible.

Le Supplément au Voyage de Bougainville approfondit d'un nouvel aspect ces considérations morales. S'ajoutant à l'épisode tahitien de l'aventure du fameux explorateur français qui avait publié en 1771 son Voyage autour du monde, il met au centre de la réflexion diderotienne la collision entre les moeurs naturelles d'un peuple dit sauvage que la civilisation n'a pas pu corrompre, et le triple code moral de l'occident, gouverné par les lois civiles et religieuses, auxquelles risque de s'opposer la nature humaine profonde, inhérente à la subsistance du corps physique. Le texte dialogique déploie le conflit entre ces deux visions du monde: le bon sauvage vit en contact avec la source de l'humanité, tandis que la civilisation européenne est en train de vivre son déclin moral. Les auteurs des Lumières, en général, aimaient bien formuler leur matière à pensée sous forme d'utopie. L'histoire insulaire sur Tahiti, avec ses moeurs sociaux et sexuels ouvertement 'libertins', est assez éloignée pour qu'elle soit lue comme du matériel abstrait, comme une curiosité. Elle a la résonnance d'une fiction, même si elle est indubitablement le récit d'une expérience réelle. Voilà encore l'art narratif de Diderot à son mieux: poétisation, dramatisation du vécu.

Orou, le Tahitien, est le porte-parole d'une sévère critique, énoncée par Diderot, par rapport à l'apparente coïncidence des trois systèmes régulateurs, trois lois différentes qui sont censées régir le comportement de l'homme, encadrer 
sa morale intime et fixer une éthique générale. Orou dissèque avec une grande simplicité la société dite incivilisée des sauvages et leur bonheur (Hartmann 2003: 259) en harmonie avec la loi naturelle en y opposant l'impossible symbiose européenne des codes civil, ecclésiastique et naturel; la collision de ces trois principes profondément hétérogènes produit nécessairement une incohérence morale:

Et où en serais-tu réduit, si tes trois maîtres, peu d'accord entre eux, s'avisaient de te permettre, de t'enjoindre et de te défendre la même chose, comme je pense qu'il arrive souvent? Alors, pour plaire au prêtre, il faudra que tu te brouilles avec le magistrat; pour satisfaire le magistrat, il faudra que tu mécontentes le grand ouvrier; et pour te rendre agréable au grand ouvrier, il faudra que tu renonces à la nature. Et sais-tu ce qui en arrivera? C'est que tu les mépriseras tous les trois, et que tu ne seras ni homme, ni citoyen, ni pieux; que tu ne seras rien; que tu seras mal avec toutes les sortes d'autorité; mal avec toi-même; méchant, tourmenté par ton coeur; persécuté par tes maîtres insensés. ${ }^{8}$

Voici le noyau de la chose: la hiérarchie sociale, le rôle disciplinaire du grand ouvrier, Dieu, l'établissement d'une autorité étatique qui devrait non seulement instaurer le fonctionnement raisonnable de la justice, mais surtout fournir au citoyen la possibilité d'une réalisation sociale qui ne serait jamais privée de dignité personnelle. Diderot propose ici un fragment de philosophie politique proto-socialiste qui laisse découvrir la nature du pouvoir ${ }^{9}$ et la conception de la démocratie qui développe ses ébauches justement dans les décennies avant la Révolution de 1789. Ce fragment, comme le suivant, ouvre une analyse percutante des conséquences dévastatrices que provoque la démocratie quand elle est réduite à un simple alibi. Diderot discute ainsi la condition du citoyen:

Si vous vous proposez d'en être le tyran, civilisez-le: empoisonnez-le de votre mieux d'une morale contraire à la nature; faites-lui des entraves de toute espèce; embarrassez ses mouvements de mille obstacles; attachez-lui des fantômes qui l'effraient; éternisez la guerre dans la caverne, et que l'homme naturel y soit toujours enchaîné sous les pieds de l'homme moral. Le voulez-vous heureux et libre? ne vous mêlez pas de ses affaires: assez d'incidents imprévus le conduiront à la lumière et à la dépravation; et demeurez à jamais convaincu que ce n'est pas pour vous, mais pour eux, que ces sages législateurs vous ont pétri et maniéré comme vous l'êtes. ${ }^{10}$

$8 \quad$ Supplément au Voyage de Bougainville, p. 556.

9 On lira ici un écho lointain de l'essai de La Boétie sur l'incapacité des peuples de se défendre contre les tyrans, Discours de la servitude volontaire (1554). 
La portée philosophique de l'écriture de Diderot est réalisée dans sa capacité exacte d'analyser les enjeux humains et sociaux, ainsi que dans les conséquences réflexives, basées sur la cohérence de la raison orientée vers le bien commun. I1 reconnaît avec une acuité accomplie les lacunes et les imperfections des systèmes mentaux et sociaux en les démasquant et en évaluant en même temps le potentiel dynamique de leur mutisme. Diderot comprend que le changement d'un système dépend de la variation de son usage, c'est-à-dire de la réalité sociale, dans laquelle ce système devrait tracer les cadres éthiques d'une communication supportable entre les gens. Il est loin de négliger la composante temporelle de ces processus: une altération systémique n'est réalisable qu'à long terme, ce qui demande un certain degré de tolérance de la part des usagers critiques - surtout si leurs tendances sont raisonnablement évolutives. La conclusion de Diderot est conciliante, légaliste, intraitable:

Nous parlerons contre les lois insensées jusqu'à ce qu'on les réforme, et en attendant nous nous y soumettrons. ${ }^{11}$

Certains textes de Diderot sont explicitement issus d'une considération philosophique (voire scientifique, dans le sens classiciste), d'autres sont empreints d'un artisme authentique. Souvent, les deux énergies créatrices se trouvent réunies en un même texte; voilà pourquoi ses courtes histoires, pour la plupart dialoguées, sont perçues comme des récits philosophiques. Le court dialogue ayant pour titre Entretien d'un philosophe avec Madame la Maréchale de **** en est un exemple élaboré. Il est souvent considéré comme de la fiction philosophique ou philosophie fictionnelle, mais il serait plus justifié d'affirmer que Diderot y combine ses attitudes philosophiques et artifices narratifs avec un tel naturel que le tout se présente comme une philosophie véridique, tissée dans les dialogues entre personnages réels. Leur potentiel communicatif est réalisé de façon à ce que ces locuteurs littéraires soient entendus comme des porteurs d'antagonismes métaphysiques.

L'entretien du personnage Diderot (par Diderot) avec la Maréchale de Broglie est une confrontation de deux visions du monde, radicalement différentes: l'épouse du Maréchal, belle comme un ange, mère de six enfants, jouit d'une vie heureuse, pragmatiquement enracinée dans la lettre des lois civiles, mais surtout religieuses; de l'autre côté un philosophe athée, réputé pour ses idées hors-piste, très critique envers les tendances moralistes du cléricalisme, et dont le bonheur consiste à soigner les vertus inhérentes afin de poursuivre le bien commun (on dirait aussi: publique) et l'impératif moral intérieur. La Maréchale, étonnée devant la stabilité intellectuelle du narrateur, questionne celui-ci comme il suit: 
- N'êtes-vous pas monsieur Crudeli?

- Oui, madame.

- C'est donc vous qui ne croyez rien?

- Moi-même.

- Cependant votre morale est d'un croyant.

- Pourquoi non, quand il est honnête homme?

- Et cette morale-là, vous la pratiquez?

- De mon mieux.

- Quoi! vous ne volez point, vous ne tuez point, vous ne pillez point?

- Très rarement. ${ }^{12}$

L'athéisme, pour Diderot, est une attitude intellectuelle qui n'ampute à personne le droit de croire ou ne pas croire, mais dénonce vigoureusement tout exclusivisme religieux quand celui-ci se mystifie à l'exagération et se réclame, au nom du mystère de la foi, d'être le seul possesseur de la vérité, réservée ainsi aux seuls receveurs d'une grâce suffisante - même si souvent inefficace. La force du philosophe, et celle de son esprit, c'est de s'opposer à la position catholique dominante en avançant que l'exclusivisme religieux n'est pas uniquement pointé vers l'extérieur, c'està-dire qu'il n'exclut pas seulement les non-croyants du cercle communicatif, mais que l'exclusion vise aussi bien le monde intérieur du croyant qui se voit ainsi privé de quelques éléments substantiels dans la compréhension du monde: le doute, le risque, l'ironie, le rire, le goût de la différence.

L'impératif moral intérieur, menant l'individu réfléchi vers la formulation du sens de la justice et du bien commun, Diderot le formule nettement quand il parle Du danger de se mettre au-dessus des lois:

Est-ce que l'homme n'est pas antérieur à l'homme de loi? Est-ce que la raison de l'espèce humaine n'est pas tout autrement sacrée que la raison d'un législateur? Nous nous appelons civilisés, et nous sommes pires que des sauvages. Il semble qu'il nous faille encore tournoyer pendant des siècles, d'extravagances en extravagances et d'erreurs en erreurs, pour arriver où la première étincelle de jugement, l'instinct seul, nous eût menés tout droit. ${ }^{13}$

Le rapport qu'une personne entretient avec sa voix intérieure, son daimon socratique, détermine le choix de l'homme soit de s'établir en tant qu'individu libre et

12 Entretien d'un philosophe avec la Maréchale de ${ }^{* * * *}$, Diderot, Oeuvres, coll. La Pléiade, Paris: Gallimard, 1996, p. 1171.

13 Entretien d'un père avec ses enfants, p. 498. 
moralement autonome, soit d'obéir inconditionnellement à n'importe laquelle des prescriptions systémiques qui lui semblent socialement pertinentes ou, tout simplement, opportunes. La conscience a une voix, tout comme l'homme à qui la phonation permet essentiellement de communiquer avec autrui et avec soi-même, c'est-à-dire de se former comme un être social. La voix de la conscience, pour Diderot, c'est la voix de l'humanité; c'est pourquoi la sonorité de la conscience est gravée sur la membrane métaphysique de la voix humaine: que celle-ci amplifie du mépris brutal, de l'indifférence fatale ou de la compréhension bienveillante, cela n'y change rien. Voilà pourquoi les contes philosophiques de Diderot n'arrêtent pas de produire des voix.

\section{BIBLIOGRAPHIE}

Attali, Jacques. Diderot ou le bonheur de penser, Paris: Fayard, 2012.

Bonnet, Jean-Claude. Diderot. Textes et débats, Paris: Librairie Générale Française, 1984.

Chouillet, Jacques. La formation des idées esthétiques de Diderot, Paris: Armand Colin, 1973.

Crocker, Lester G. «Le Neveu de Rameau, une expérience morale», C.A.I.E.F. 13, 1961: p. 133-155.

Delon, Michel (éd). Album Diderot, coll. La Pléiade, Paris: Gallimard, 2004.

Didier, Béatrice. «N'êtes-vous pas Monsieur Diderot?», Diderot: Contes, Paris: Librairie Générale Française, 2010: p. 5-34.

Fontenay, Elisabeth de. Diderot ou le matérialisme enchanté, Paris: Grasset, 1981. Hartmann, Pierre. Diderot, la figuration du philosophe. Paris: José Corti, 2003. Mornet, Daniel. Diderot, coll. Connaissance des lettres, Paris: Hatier, 1966.

Pérol, Lucette. «Introduction», Diderot: Contes et entretiens, Paris: Flammarion, 1977: p. 13-37.

Proust, Jacques. Lectures de Diderot, coll. U2, Paris: Armand Colin, 1974.

Stenger, Gerhardt. Diderot. Le combattant de la liberté, Paris: Perrin, 2013.

Versini, Laurent. «Introduction», Diderot: Oeuvres, tome II, Contes, Bouquins, Paris: Robert Laffont, 1994, p. 1-15.

Wilson, Arthur M. Diderot. Sa vie et son oeuvre, Paris: Laffont/Ramsay, 1985. 


\section{Govorna izmenjava v Diderotovih filozofskih zgodbah}

Diderotove filozofske zgodbe, kakor tudi večina njegovih romanov, delujejo kot latentno gledališče, ker je premi govor njihova ključna sestavina. Diderot v svojih proznih besedilih združuje oralne sestavine literarne pripovedi s formulacijo filozofske misli. Za humanista ni nič presenetljivo, če skuša do svoje interpretacije sveta priti po dveh vzporednih poteh. Prav spričo svojstvene izpeljave te dvojnosti - refleksivne in narativne - se je Diderot uveljavil kot eden najbolj dialoških pisateljev in filozofov evropskega osemnajstega stoletja. Ti dve ustvarjalni energiji sta najizraziteje prepleteni v kratkih zgodbah, napisanih med letoma 1768 in 1774. Te literarne pripovedi določa močna filozofska komponenta, ker se vztrajno odpirajo $\mathrm{k}$ priobčevanju temeljnih etičnih dilem: kadar se je treba soočiti $\mathrm{z}$ arbitrarnostjo zakonov ali poljubnostjo javnega mnenja, besedo dajejo posamezniku.

Ključne besede: govorna izmenjava, Diderot, etika govora, dialog, literarni glas

\section{Speech Exchange in Short Philosophical Stories by Diderot}

Diderot's philosophical stories, as most his novels, work as potential theatre, since dialogue is their main narrative structure. In his prose works, Diderot produces a fusion of speech mechanisms and philosophical arguments: there is more than one way for a humanist to formulate the interpretations of what he perceives as critical. The singularity of his narrative (reflective and story-telling) makes of Diderot one of the most accomplished dialogue writers in the 18th century. These two creative energies are the real tissue of Diderot's short stories, written from 1768 to 1774 . The stories are defined by their philosophical component, communicating persistently the ethical dilemmas: when it comes to confronting legal arbitrarity or public opinion, the word is always given to the individual.

Keywords: speech exchange, Diderot, speech ethics, dialogue, literary voice 